\title{
POLARIMETRIC-INTERFEROMETRIC BOREAL FOREST SCATTERING MODEL FOR BIOMASS END-TO-END SIMULATOR
}

\author{
Maciej J. Soja ${ }^{l)}$ and Lars M. H. Ulander ${ }^{1,2)}$ \\ ${ }^{1)}$ Chalmers University of Technology, Gothenburg, Sweden \\ ${ }^{2}$ Swedish Defence Research Agency, Linköping, Sweden
}

\begin{abstract}
A polarimetric-interferometric forward model (FM) for extended covariance matrix modeling is presented. The FM has been designed to be used within the end-to-end simulator for BIOMASS, a new ESA satellite mission aiming at the global mapping of above-ground forest biomass with P-band synthetic aperture radar (SAR). The FM uses linear regression models for prediction of backscatter intensity and HH-VV correlation coefficient, and the random volume over ground (RVoG) model for the prediction of the interferometric correlation coefficients. For boreal forest, parameter values for these sub-models have been derived using polarimetric-interferometric SAR data acquired within the BioSAR 2007 campaign over the Swedish test site Remningstorp. The FM is evaluated qualitatively in a boreal forest scenario through a side-byside comparison with BioSAR 2007 data. The general agreement is good, although there are regions with structures which cannot be reproduced by the model, probably due to insufficient forest description by the input parameters.
\end{abstract}

Index Terms - BIOMASS, forward model, extended covariance matrix

\section{INTRODUCTION}

In May 2013, European Space Agency (ESA) selected the BIOMASS satellite for the 7th Earth Explorer mission. The main goal of the mission is accurate, high-resolution mapping of global forest resources in terms of above-ground biomass (total mass of living forest tissue), biomass change, and forest height. This will, in turn, aid global carbon cycle modelling, and eventually lead to improved climate change predictions [1].

BIOMASS will feature the first P-band synthetic aperture radar (SAR) in space, and also the lowest frequency SAR in space. The main advantage of $\mathrm{P}$-band radar is its penetration capabilities. In forestry, this means that a P-band radar has the capability to see through the canopy and it is sensitive to scattering from trunks and large branches, which is where most biomass is stored. These structures are also significantly more stable in time (compared to the canopy), which means that temporal decorrelation at P-band is relatively low, and repeat-pass, multi-baseline interferometry and tomography will routinely be carried out. Also, with the fully polarimetric capabilities of BIOMASS, estimation of forest height will be done from polarimetricinterferometric SAR (PolInSAR) data.

In order to be able to evaluate the performance of the future BIOMASS satellite, a BIOMASS end-to-end simulator (BEES) has been implemented for both boreal and tropical forests [2]. Using the simulator, system effects can be modeled, and error budgets can be estimated. An important part of BEES is the forward model, which predicts the extended covariance matrix for different forest biomes from a small number of input parameters. A preliminary version of the model has been presented in [3]. In this paper, the boreal forest version of the forward model will be presented in its final version, and its performance in 2D modelling will be assessed qualitatively on data from BioSAR 2007.

\section{DATA}

SAR data were acquired with a flight heading of $200^{\circ}$ over Remningstorp, a hemi-boreal test site located in southern Sweden, by the airborne ESAR system in May 2007 during BioSAR 2007 [4]. Small-footprint lidar-based estimates of biomass and forest height for 58 forest stands have been used for the estimation of model parameters. The errors of the FM have been estimated using ten $80 \mathrm{~m} \times 80 \mathrm{~m}$ forest plots, for which stem diameter has been measured for all trees, and height for a subset of trees [5]. For quantitative performance analysis, biomass and forest height maps derived from lidar data and species stratification information are used as input to the FM.

\section{FORWARD MODEL}

The model is designed to compute the extended covariance matrix for a polarimetric-interferometric pair. First, it is assumed that the backscatter signature is equal for both the master and slave images, which gives the following extended covariance matrix:

$$
\hat{C}_{6}=\left[\begin{array}{cc}
\widehat{V} & \widehat{K}_{12} \\
\widehat{K}_{12}^{H} & \widehat{V}
\end{array}\right]
$$

where $H$ is the Hermitian (conjugate transpose) operator, and $\widehat{V}$ is the polarimetric covariance matrix, formulated as:

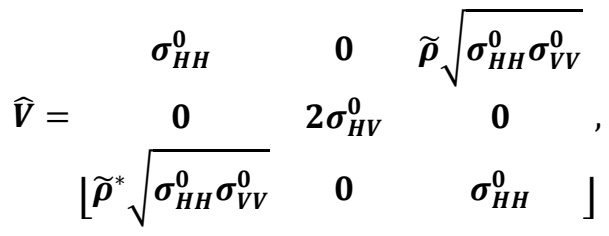

(C) 2014 IEEE. Personal use of this material is permitted. Permission from IEEE must be obtained for all other uses, in any current or future media, including reprinting/republishing this material for advertising or promotional purposes, creating new collective works, for resale or redistribution to servers or lists, or reuse of any copyrighted component of this work in other works. 
where the correlation between co- and cross-polarized channels has been shown to be zero for monostatic acquisitions [6].

The polarimetric-interferometric covariance matrix $\widehat{K}_{12}$ can be formulated as:

$$
\widehat{\boldsymbol{K}}_{12}=\left[\begin{array}{ccc}
\widetilde{\gamma}_{H H} \sigma_{H H}^{0} & \mathbf{0} & \widetilde{\rho} D \\
\mathbf{0} & 2 \widetilde{\gamma}_{H V} \sigma_{H V}^{0} & \mathbf{0} \\
(\widetilde{\rho} D)^{*} & \mathbf{0} & \widetilde{\gamma}_{V V} \sigma_{V V}^{0}
\end{array}\right]
$$

where

$$
D=\frac{\left(\widetilde{\gamma}_{H H}+\widetilde{\gamma}_{V V}\right)}{2} \sqrt{\sigma_{H H}^{0} \sigma_{V V}^{0}}
$$

Backscattering coefficient $\left(\sigma^{\circ}\right)$ for polarization PQ is modelled in $\mathrm{dB}$ using a linear model with an additive error:

$$
\begin{aligned}
{\left[\sigma_{P Q}^{0}\right]_{d B}=a_{P Q}+} & b_{P Q} \log _{10} B+10 \log _{10}\left(\cos \theta_{i}\right) \\
& +N\left(0, s_{P Q}^{2}\right),
\end{aligned}
$$

where $\theta_{i}$ is the local angle of incidence, $B$ is the biomass in tons $(\mathrm{Gg})$ per hectare $(100 \mathrm{~m} \times 100 \mathrm{~m})$, and the last term is a normally distributed, zero-mean error, with standard deviation estimated using the 10 field plots. The parameter values estimated for the boreal data can be found in Table 1 .

Table 1 Parameter values for backscatter model.

\begin{tabular}{|l|l|l|l|}
\hline Polarization & \multicolumn{1}{|c|}{$a_{P Q}$} & \multicolumn{1}{|c|}{$b_{P Q}$} & $S_{P Q}$ \\
\hline HH & -20.1 & 8.1 & 1.3 \\
\hline HV & -20.7 & 4.2 & 0.7 \\
\hline VV & -6.7 & 0.6 & 1.2 \\
\hline
\end{tabular}

The complex correlation coefficient between the $\mathrm{HH}$ and VV channels $\tilde{\rho}$ is for the boreal scenario modelled as:

$$
\tilde{\rho}=\left(0.39+N\left(0,0.07^{2}\right)\right) \cdot e^{i\left(-41.5^{\circ}-0.27 B+N\left(0,11.6^{2}\right)\right)}
$$

where the last term in both magnitude and phase are normally distributed, zero-mean errors. The standard deviations have been estimated from the same field plots.

For the interferometric part, correlation coefficients $\left(\tilde{\gamma}_{P Q}\right)$ are modeled using the random volume over ground (RVoG) model with two different profile functions. Here, the exponential profile will be used, yielding:

$$
\tilde{\gamma}_{v o l}=\frac{\int_{0}^{h_{\text {top }}} f(z) e^{i k_{z} z} d z}{\int_{0}^{h_{t o p}} f(z) d z}=\frac{1}{1+\frac{i k_{z} \cos \left(\theta_{i}\right)}{2 \sigma}} \cdot \frac{e^{\left(\frac{2 \sigma}{\cos \left(\theta_{i}\right)}+i k_{z}\right) h_{t o p}}-1}{e^{\frac{2 \sigma h_{t o p}}{\cos \left(\theta_{i}\right)}}-1},
$$

where $\sigma$ is the extinction coefficient, $h_{t o p}$ is top forest height, and $k_{z}$ is the vertical wave number. This is inserted in the general $\mathrm{RVoG}$ expression giving:

$$
\tilde{\gamma}_{P Q}=\exp \left(i h_{0} k_{z}\right) \cdot \frac{\tilde{\gamma}_{v o l} \cdot \gamma_{t e m p}+\mu_{P Q}}{1+\mu_{P Q}},
$$

where $\gamma_{\text {temp }}=e^{-\frac{B_{T}}{\tau_{D}}}$ is a temporal decorrelation term, $B_{T}$ is the temporal baseline, $\tau_{D}$ is decorrelation time, $h_{0}$ is ground height, and $\mu_{P Q}$ are ground-to-volume ratios.

In the boreal forest model, $\sigma=N\left(0.1,0.1^{2}\right) \mathrm{dB} / \mathrm{m}$ has been chosen, based on results from PolInSAR height inversion, and $\mu_{H H}=N\left(6.4,1.3^{2}\right) \mathrm{dB}, \mu_{H V}=N\left(-2.1,0.7^{2}\right) \mathrm{dB}$, and $\mu_{V V}=N\left(2.2,0.7^{2}\right) \mathrm{dB}$ were estimated from the data using polarimetric decomposition. $h_{0}, h_{\text {top }}, k_{z}, B_{T}$, and $\theta_{i}$ are known input parameters. $\tau_{D}$ is set through the choice of temporal decorrelation scenario.

\section{RESULTS}

The forward model is evaluated qualitatively for $2 \mathrm{D}$ mapping. Predictions of $\sigma_{P Q}^{0}, \tilde{\rho}$, and $\tilde{\gamma}_{P Q}$ are made from biomass map, forest height map, and DTM, and compared to ESAR data. Temporal decorrelation is neglected. The results are shown in $0,0,0$, and Figure 4 . The results are in general good, but in the case of VV-backscatter, HH-VV coherence, and interferometric coherences, the model does not predict some spatial changes, probably due to insufficient description of the scene with the input data. Information on, e.g., forest density or forest type would probably improve modeling.

\section{REFERENCES}

S. Quegan, T. Le Toan, J. Chave, J. Dall, K. P. Papathanassiou, F. Rocca, et al., "BIOMASS, Report for Mission Selection," European Space Agency (SP1324/1)2012.

P. Lopez-Dekker, F. De Zan, T. Borner, M. Younis, K. Papathanassiou, T. Guardabrazo, et al., "BIOMASS endto-end mission performance simulator," in IEEE International Geoscience and Remote Sensing Symposium (IGARSS), Vancouver, BC, Canada, 2011, pp. 4249-4252.

[3] M. J. Soja and L. M. H. Ulander, "A Hybrid Model for Interferometric and Polarimetric P-band SAR Modelling of Forests," in Proceedings of PolInSAR 2011, 2011.

[4] I. Hajnsek, R. Scheiber, L. Ulander, A. Gustavsson, G. Sandberg, S. Tebaldini, et al., "BioSAR 2007 technical assistance for the development of airborne SAR and geophysical measurements during the BioSAR 2007 experiment: Final report without synthesis," ESA contract no. 20755/07/NL/CB2008.

G. Sandberg, L. M. H. Ulander, J. E. S. Fransson, J. Holmgren, and T. Le Toan, "L- and P-band backscatter intensity for biomass retrieval in hemiboreal forest," Remote Sensing of Environment, vol. 115, pp. 28742886, 2011

S. V. Nghiem, S. H. Yueh, R. Kwok, and F. K. Li "Symmetry Properties in Polarimetric Remote Sensing," Radio Science, vol. 27, pp. 693-711, 1992. 

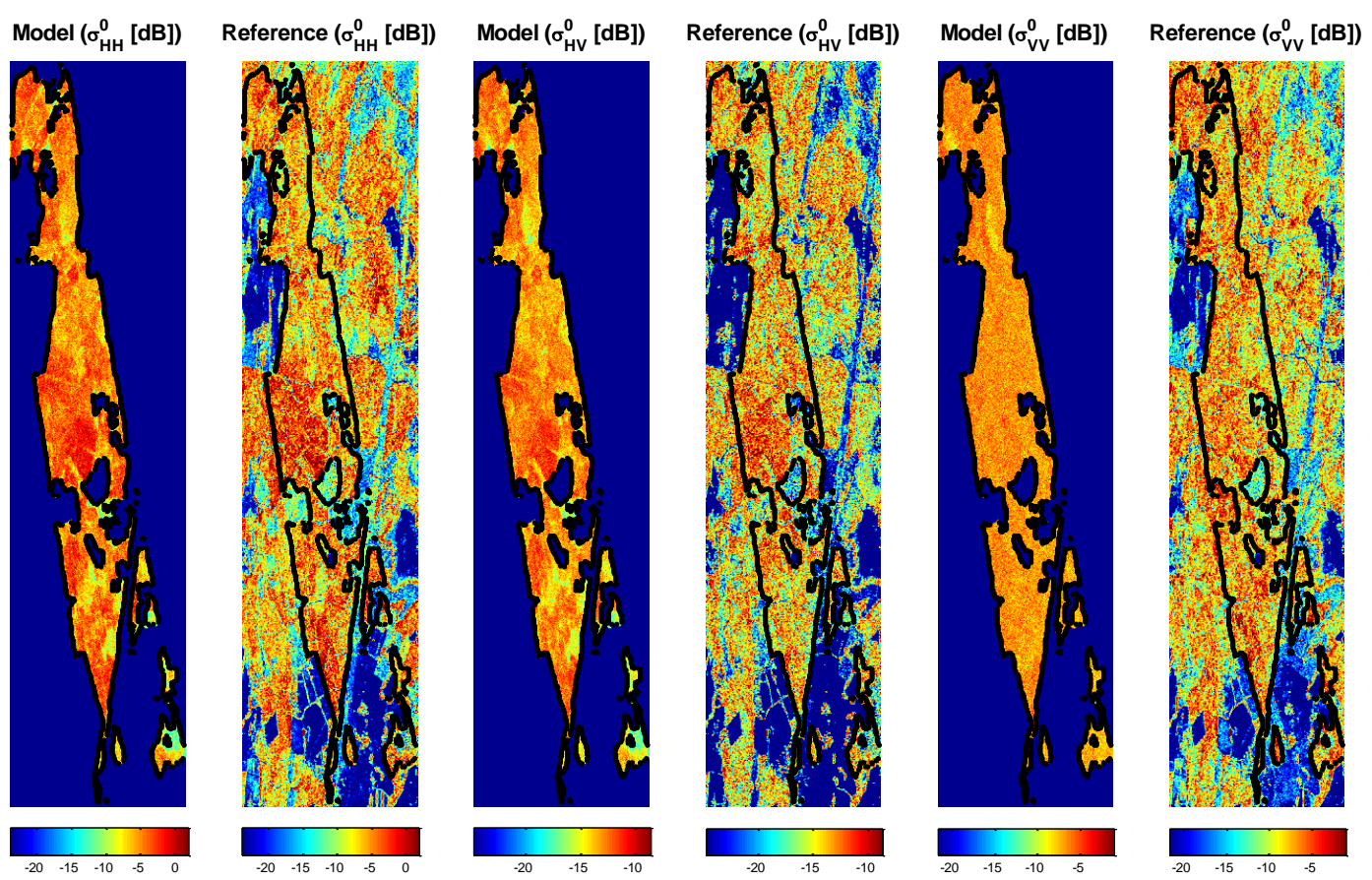

Figure 1 Modeling results for backscattering coefficient compared to ESAR data. All three polarizations are shown. The black outline marks the largest region covered by all required input data. For $\mathbf{H H}$, the scale range is $\mathbf{- 2 3} \mathrm{dB}$ to $2 \mathrm{~dB}$, for $\mathrm{HV}$, the scale range is $-25 \mathrm{~dB}$ to $-8 \mathrm{~dB}$, and for $\mathrm{VV}$, the scale range is $-21 \mathrm{~dB}$ to $-1 \mathrm{~dB}$.
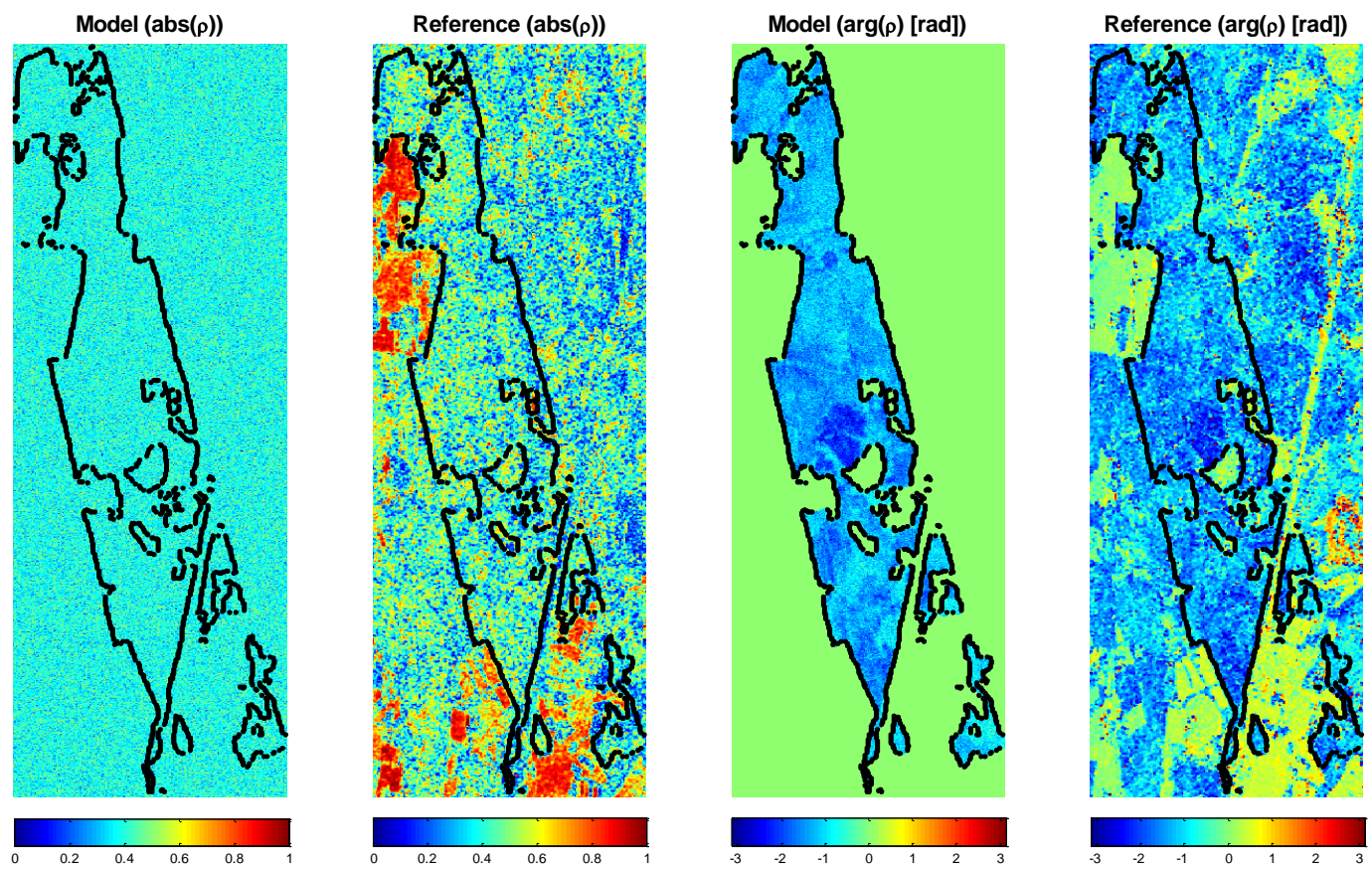

Figure 2 Modeling results for polarimetric coherence and phase compared to ESAR data. The black outline marks the largest region covered by all required input data. The scale range is 0 to 1 for coherence and $-\pi$ to $\pi$ for phase. 

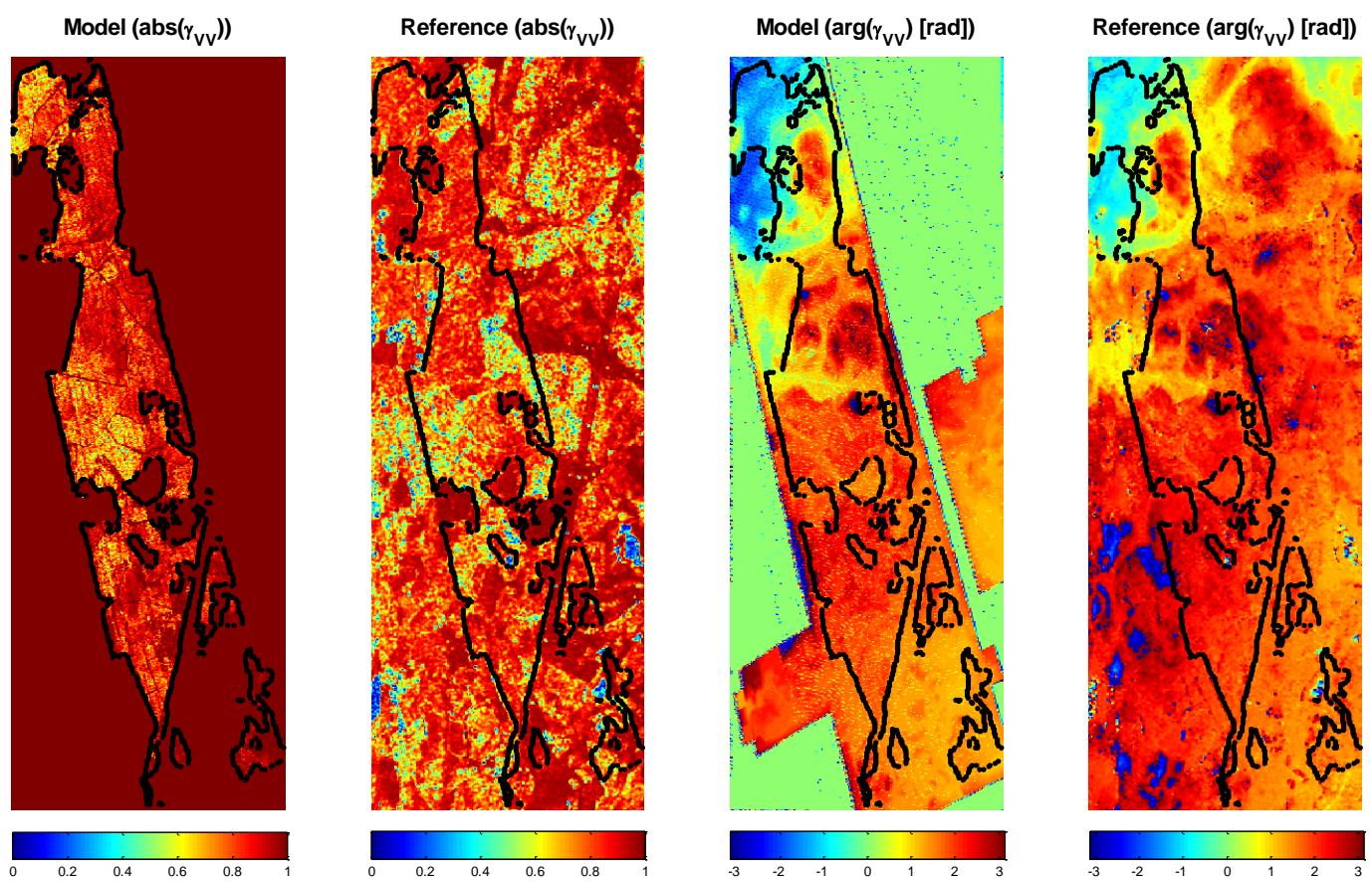

Figure 3 Modeling results for interferometric coherence and phase in comparison with ESAR data. VV-polarization is shown here. The scale range is 0 to 1 for coherence and $-\pi$ to $\pi$ for phase.
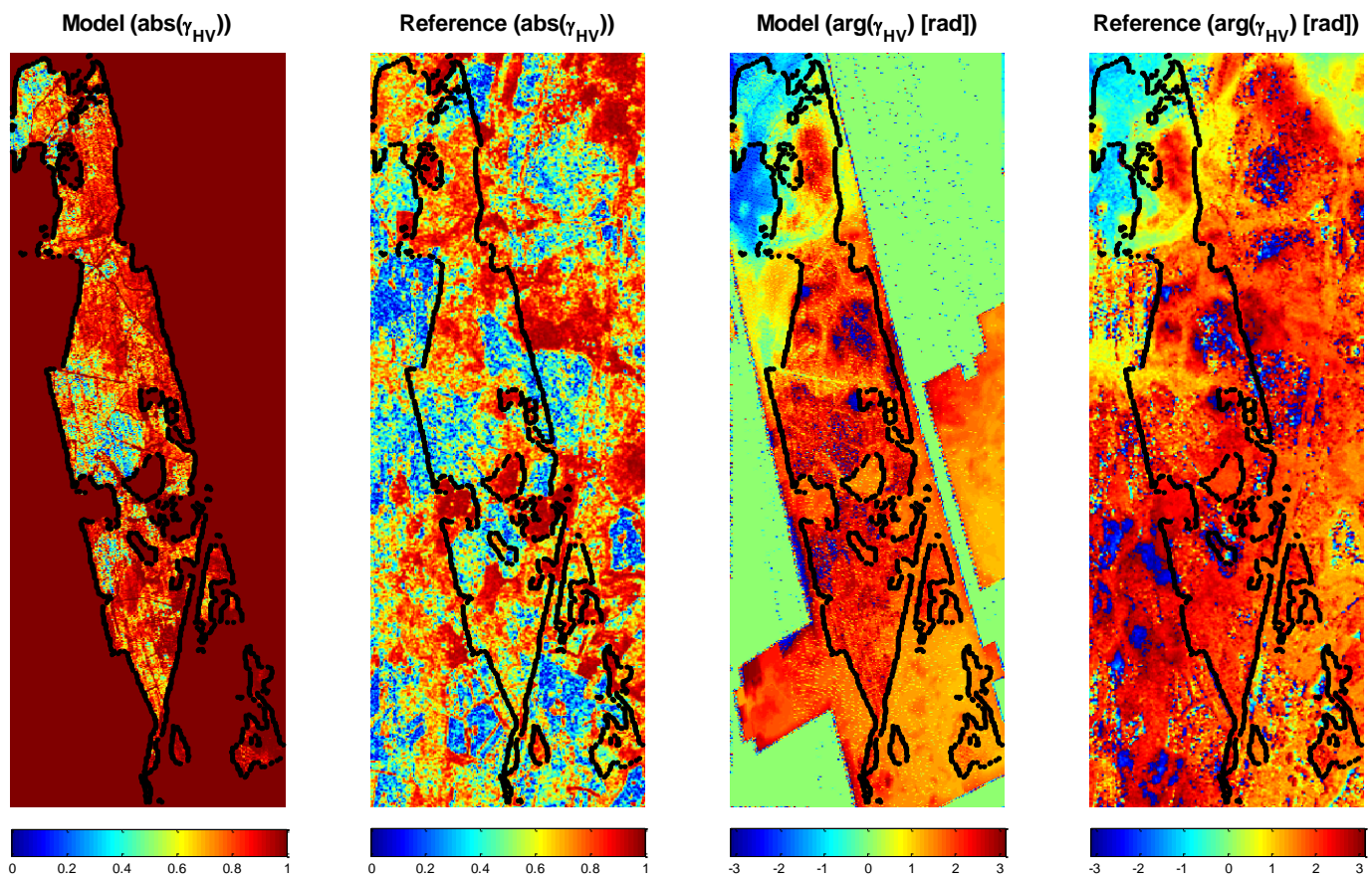

Figure 4 Modeling results for interferometric coherence and phase in comparison to ESAR data. HV-polarization is shown here. The scale range is 0 to 1 for coherence and $-\pi$ to $\pi$ for phase. 